\title{
ASYMPTOMATIC ANOMALOUS LEFT CORONARY ARTERY FROM THE PULMONARY ARTERY (ALCAPA) IN AN ADULT.
}

\author{
Demetrio Sharp Dimitri ${ }^{1}$, Andrew Panakos ${ }^{2}$, Carlos Alfonso ${ }^{3}$, and Vicente Orozco-Sevilla ${ }^{2}$ \\ ${ }^{1}$ University of Cincinnati Academic Health Center \\ ${ }^{2}$ University of Miami Hospital and Clinics \\ ${ }^{3}$ University of Miami, Miller School of Medicine
}

January 12, 2021

\begin{abstract}
We describe a patient in whom this anomaly was diagnosed during cardiac catheterization for evaluation of progressive shortness of breath and mild to moderate mitral regurgitation founded on a transthoracic echocardiogram.A 58-year-old hypertensive female was evaluated for progressive shortness of breath. Her physical examination showed a regular heart rate and rhythm, with normal heart sounds with a holosystolic systolic murmur on the 4th intercostal midclavicular line on auscultation. Electrocardiography showed normal sinus rhythm and criteria and possible left ventricular hypertrophy. Stress Echocardiogram showed with no evidence of ischemia preserved left ventricular function with an ejection fraction of 55-60\%, with mild to moderate mitral regurgitation. Patient underwent a coronary catheterization for persistent shortness of breath which indicated no evidence of obstructive coronary disease, normal left ventricular function with mild to moderate mitral insufficiency. Angiography of the right coronary artery showed a very large and tortuous artery that collateralizes into the left main system and supplies the entire left coronary artery system. The left main coronary origin was from the pulmonary artery. CTA confirmed the same findings. The recommendations between cardiology and cardiothoracic surgery were to maximize medical therapy and follow up due to lack of myocardial ischemia or severe valvular disease. Currently there are no guidelines or gold standard management for patients with adult form of ALCAPA that present without ischemic burden or for patients with the disease that present asymptomatic with significant coronary collateralization.
\end{abstract}

Title:

Asymptomatic Anomalous Left Coronary Artery from the Pulmonary Artery (ALCAPA) in an Adult.

Authors:

Demetrio Sharp Dimitri MD ${ }^{1}$, Andrew Panakos MD², Carlos E Alfonso MD², Vicente Orozco-Sevilla MD ${ }^{1}$. Institution:

University of Cincinnati Medical Center, Department of Internal Medicine. ${ }^{1}$

University of Miami Health, Department of Cardiovascular Disease. ${ }^{2}$

Corresponding Author:

Demetrio Sharp Dimitri, MD

Department of Internal Medicine 
University of Cincinnati Medical Center, Department of Internal Medicine

Email: sharpddo@ucmail.uc.edu

Disclosure:

Demetrio Sharp Dimitri MD: None.

Andrew Panakos MD: None.

Carlos E Alfonso MD: None.

Vicente Orozco-Sevilla MD: None

Word count: 629

Keywords: ALCAPA, Mitral valve regurgitation, congenital cardiac defect, coronary anomaly Summary

Anomalous origin of the left coronary artery from the pulmonary artery (ALCAPA) is considered a rare and very fatal congenital coronary artery anomaly. It is estimated that this congenital abnormality affects 1 of every 300,000 live births and accounts for $0.25 \%$ of all congenital heart defects worldwide. ALCAPA manifests itself most commonly in infant population and it has a 1-year mortality rate of approximately $90 \%$, therefore it is not often considered a diagnosis in the adult population $(4,5)$. When it presents in adult it is considered an important cause of sudden cardiac death $(1,2)$. We describe a patient in whom this anomaly was diagnosed during cardiac catheterization for evaluation of progressive shortness of breath and mild to moderate mitral regurgitation founded on a transthoracic echocardiogram (3).

\section{CASE}

A 58-year-old otherwise hypertensive female that was evaluated because of her progressive shortness of breath and mild to moderate mitral regurgitation founded on a transthoracic echocardiogram.

Her physical examination showed a regular heart rate and rhythm, with normal heart sounds with an II/III holosystolic systolic murmur on the $4^{\text {th }}$ intercostal midclavicular line, no rubs or gallops were present on auscultation. No lower extremity pitting edema was noticed.

Electrocardiography showed normal sinus rhythm and criteria and possible left ventricular hypertrophy.

Stress Echocardiogram with no evidence of ischemia and preserved left ventricular function with an ejection fraction of 55-60\%, left atrium dilated with mild to moderate mitral regurgitation.

Patient underwent a coronary catheterization which indicated no evidence of obstructive coronary disease, normal left ventricular function with mild to moderate mitral insufficiency. Angiography of the right coronary artery showed a very large and tortuous artery that collateralizes into the left main system and supplies the entire left coronary artery system. The left main coronary origin was from the pulmonary artery. CTA confirmed the same findings.

The recommendations between cardiology and Cardio Thoracic surgery were to maximize medical therapy including beta blockers and diuresis and close follow up due to the lack of myocardial ischemia,severe valvular disease or heart failure.

\section{Discussion.}

ALCAPA is a very rare condition in which the left main coronary artery originates on the pulmonary artery. The decreased flow into the left coronary arteries due to coronary artery steal from the low pulmonary artery resistance leads to ischemia and infarction of the myocardial tissue an usually is lethal if is not corrected as an infant (3). 
The infant type of ALCAPA is considered to be very fatal if untreated with a 1-year mortality of approximately $90 \%$ (5). Infants commonly present with failure to thrive, breathing difficulties, irritability, and distress while crying or eating. The adult type of ALCAPA is related to sudden cardiac death and symptoms of ischemia, mitral valve regurgitation, and left ventricular dysfunction $(3,4)$.

The treatment of the ALCAPA infant type is absolutely surgically with either two-coronary system coronary button transfer, Takeuchi procedure (trans- pulmonary baffling), coronary artery bypass grafting (CABG) combined with ligation of the anomalous left coronary artery $(4,5)$. ALCAPA Adult type treatment is sometimes controversial, although the preferred approach is surgical including CABG combined with anomalous left coronary artery ligation and patch of the pulmonary artery $(4,5)$. This approach should only be considered if the patient adult has or develops evidence of ischemia, severe mitral regurgitation or heart failure secondary to a significant shunt and volume overload (4).

Currently there are no guidelines or gold standard management for patients with adult form of ALCAPA that present with minimal to non-disease of the myocardium? How can we monitor the disease course and possibly risk stratify patients for possible complications? Is there a screening benefit for patients with high probability of ALCAPA? How much real benefit does the surgical intervention really offers to this patient's population group? What type of conduit is appropriate if CABG planned vein or double mammary. This case raises questions regarding the management of this rare congenital coronary anomaly

\section{Reference:}

1.- Alexi-Meskishvili V, Berger F, Weng Y, lange pE, Hetzer R. anomalous origin of the left coronary artery from the pulmonary artery in adults. J Card surg 1995; 10: 309-315.

2.- Arciniegas E, Farooki ZQ, Hakimi M, Green EW. Management of anomalous left coronary artery from the pulmonary artery. Circulation 1980;62(2 pt 2):I180-I189.

3- Fernandes ED, Kadivar H, Hallman GL, Reul GJ, Ott DA, Cooley DA. Congenital malformations of the coronary arteries: the Texas Heart Institute ex- perience. Ann Thorac Surg 1992;54:732-740.

4.- H. Wesselhoeft, J.S. Fawcett, A.L. Johnson. Anomalous origin of the left coronary artery from the pulmonary trunk: Its clinical spectrum, pathology, and pathophysiology, based on a review of 140 cases with seven further cases. Circulation, 38 (1968), pp. 403-425.

5.- Ishihata T, Takeda H, Katohno E, et al. An adult case of Bland-White-Garland syndrome with collaterals from the bronchial artery. Heart Vessels 1994;9:218-222.

IMAGE 1; 


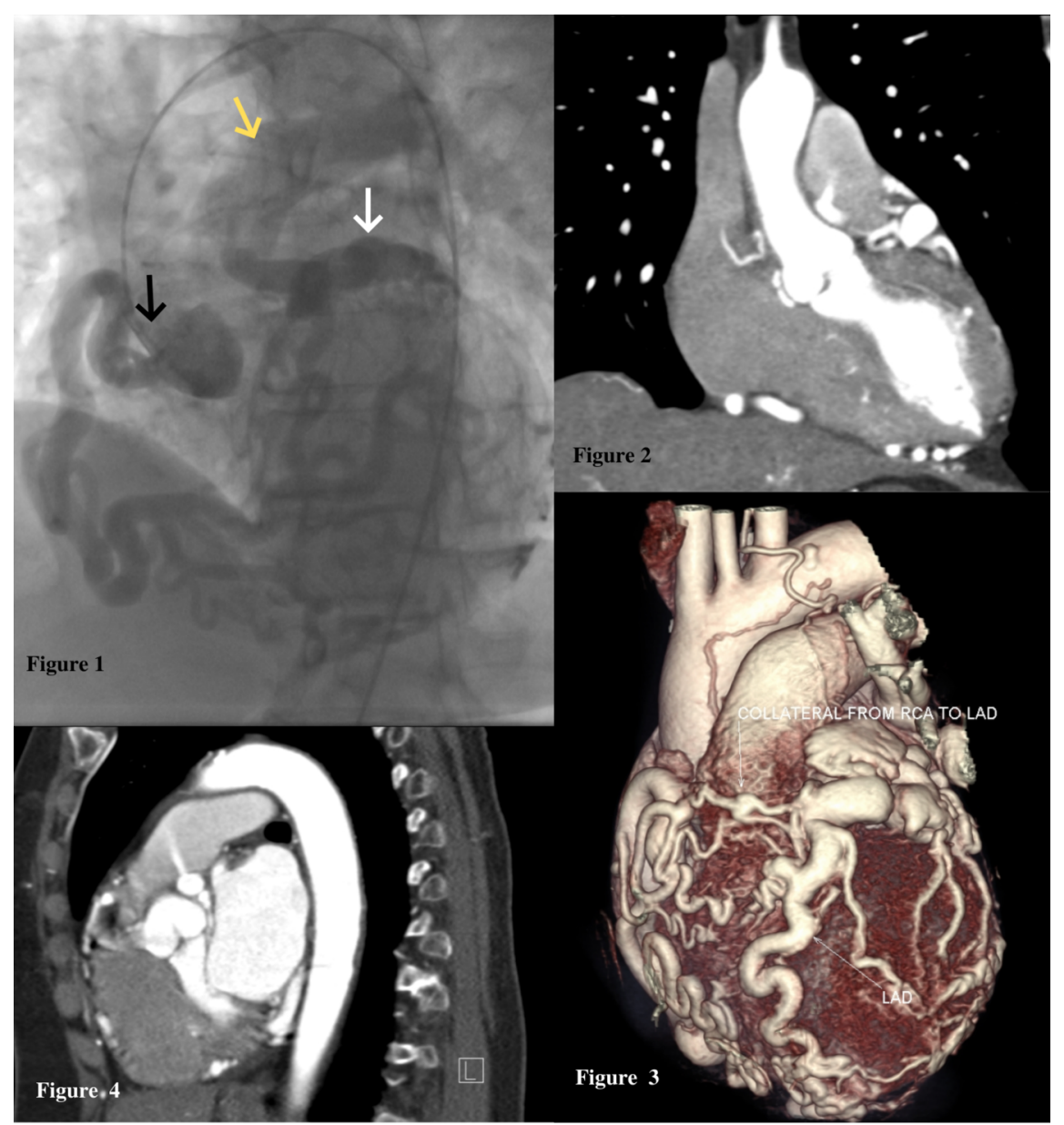

Figure 1: Coronary angiogram obtained with a single RCA injection showing a tortuous and dilated RCA (Black Arrow) as well as an equally tortuous and dilated left coronary system (White Arrow). Contrast reaches the main pulmonary artery because of the pathologic steal phenomenon (Yellow Arrow). Figure 2: Chest CT. Evidencing opacification within the left coronary artery. Retrograde filling of left cor coronary artery emerges from pulmonary artery trunk. Figure 4: Coronary CT. Anomalous left coronary artery originating from the
pulmonary artery. 


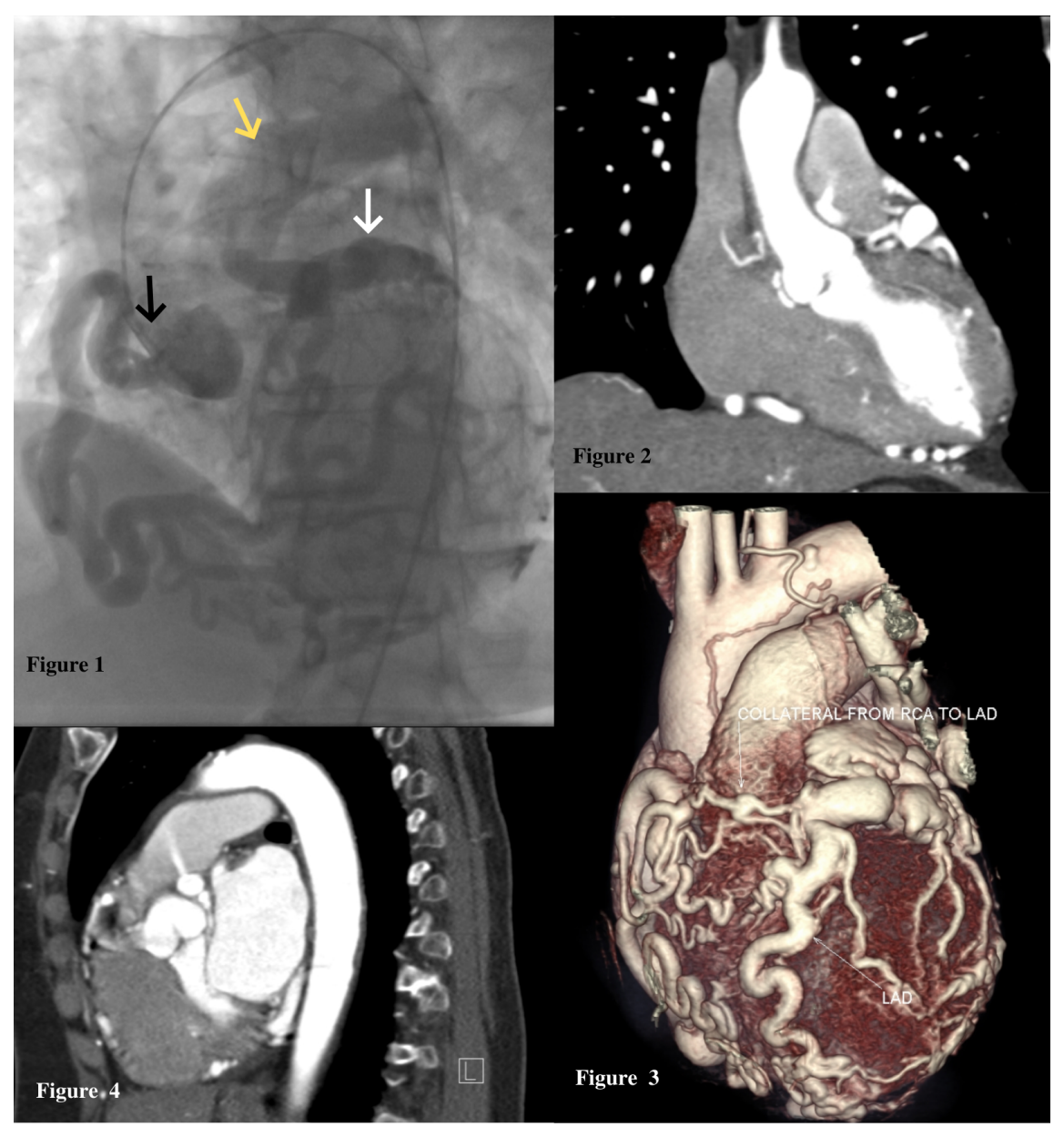

Figure 1: Coronary angiogram obtained with a single RCA injection showing a tortuous and dilated RCA (Black Arrow) as well as an equally tortuous and dilated left coronary system (White Arrow). Contrast reaches the main pulmonary artery because of the pathologic steal phenomenon (Yellow Arrow). Figure 2: Chest CT. Evidencing opacification within the left coronary artery. Retrograde filling of left coronary artery circulation. Figure 3: 3-D CT Angiogram. RCA with a large number of collaterals feeding the left coronary. Left coronary artery emerges from pulmonary artery trunk. Figure 4: Coronary CT. Anomalous left coronary artery originating from the pulmonary artery. 